\title{
Effective Field Theory and Nuclear Mean-Field Models
}

\author{
R. J. Furnstahl ${ }^{\mathrm{a} *}$ and Brian D. Serot ${ }^{\mathrm{b} \dagger}$ \\ ${ }^{a}$ Department of Physics, Ohio State University, Columbus, OH 43210 \\ ${ }^{\mathrm{b}}$ Department of Physics, Indiana University, Bloomington, IN 47405
}

The implications of an effective field theory (EFT) interpretation of nuclear mean-field phenomenology are reviewed.

Recent work has demonstrated that effective field theory (EFT) concepts and methods can explain the successes and limitations of mean-field models of nuclear structure and reactions [1 5]. For example, coefficients in successful relativistic (QHD) and nonrelativistic (Skyrme) mean-field models are consistent with naive dimensional analysis (NDA) and naturalness, as expected in low-energy effective field theories of QCD [6].

NDA implies an expansion of the mean-field energy density in $\rho / f_{\pi}^{2} \Lambda$ with coefficients of order unity ("naturalness"), where $\rho$ is the nuclear density, $f_{\pi} \approx 93 \mathrm{MeV}$ is the piondecay constant, and $\Lambda$ is the scale of non-Goldstone boson physics (500 to $1000 \mathrm{MeV}$ ) [1.33]. This expansion parameter is between $1 / 7$ and $1 / 4$ at equilibrium nuclear density. Nuclear matter binding energies verify this expansion for all models that fit nuclear data accurately. In addition, truncation errors due to omitted terms are predicted.

In Fig. 团, individual contributions to the energy per particle from terms in the energy functional of two general QHD models are plotted against the net power of the mean meson fields. In an EFT, one expects all terms consistent with underlying symmetries, excluding redundancies that can be removed by redefining the fields. The models shown include all terms in the expansion implied by NDA with coefficients determined by a $\chi^{2}$-type optimization to bulk properties of magic nuclei [2]. The crosses are expected values from NDA estimates, with the error bars spanning a reasonable range of natural coefficients (from $1 / 2$ to 2 ). One model is to the left of each cross and one is to the right. The hierarchy of contributions predicted by NDA is manifest.

In Fig. 2, the impact of different model truncations is shown by plotting $\chi^{2}$ against the maximum power of fields. The "full" models (which include all terms at a given order) show that one needs to go to the fourth power of fields to get excellent fits, but no further. The " $\phi^{n}$ only" results, with only scalar fields for $n>2$, show that nearly optimal results can be obtained with just a subset of terms at each order. Thus the parameters are underdetermined by the data. These features explain the successes of the most widely used QHD models, which add only $\phi^{3}$ and $\phi^{4}$ terms to the original Walecka model.

${ }^{*}$ Supported in part by NSF grants PHY-9511923 and PHY-9800964.

†Supported in part by the DOE under Contract No. DE-FG02-87ER40365. 


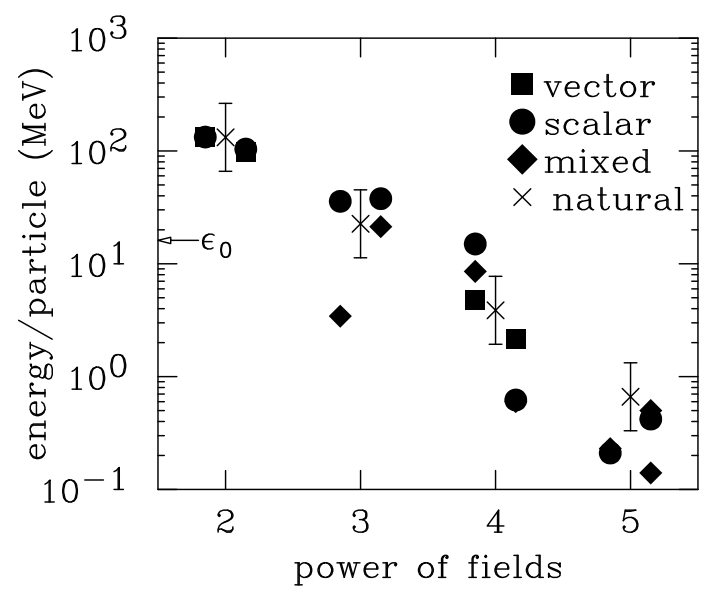

Figure 1. Energy contributions to two QHD models, with estimates based on NDA and natural coefficients (error bars).

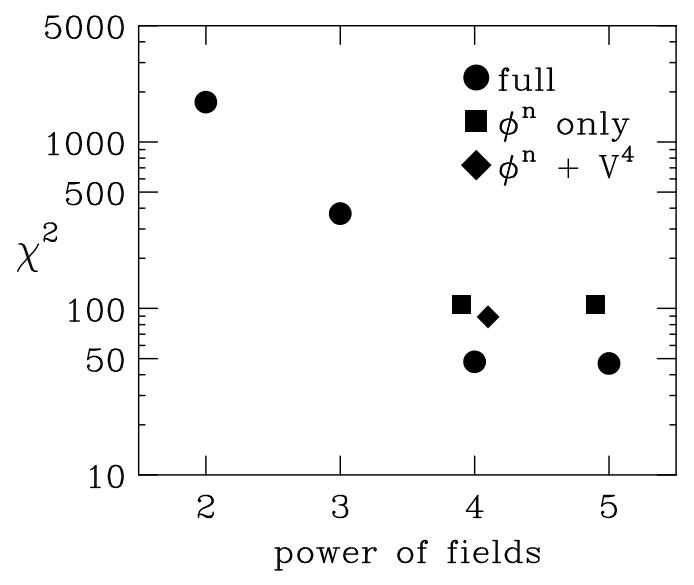

Figure 2. $\chi^{2}$ values for QHD meson models, according to the truncation level.

It is often said that since QHD models use local nucleon fields, they do not incorporate the effects of nucleon compositeness. Indeed, quark-meson coupling (QMC) models were introduced to remedy this "deficiency" [7]. Unlike QHD models, QMC models have density- or field-dependent couplings, e.g., $g_{s} \sigma \rightarrow g_{s}(\sigma) \sigma$. However, the EFT perspective shows the QMC models are in fact a subset of the general QHD models. A simple field redefinition, $g_{s}(\sigma) \sigma \bar{N} N \rightarrow g_{s} \sigma^{\prime} \bar{N} N+a \sigma^{\prime 3}+b \sigma^{\prime 4}+\cdots$, moves the QMC vertex corrections into meson propagator corrections already contained in QHD models. Observables cannot depend on these off-shell manipulations.

In addition, general QHD models incorporate single-nucleon form factors explicitly in a derivative expansion [2]. Low energy means low resolution, which implies that a derivative expansion is efficient. This is analogous to studying a complicated charge distribution using long wavelengths. One may be sensitive to the dipole moment, and any convenient model (preferably with a systematic expansion) that reproduces the moment will work. However, fitting the dipole moment does not imply that the quadrupole moment will be correct! Similarly, extrapolations of mean-field models to uncalibrated regions are dangerous. A virtue of the EFT approach is that the limits of the model are well understood.

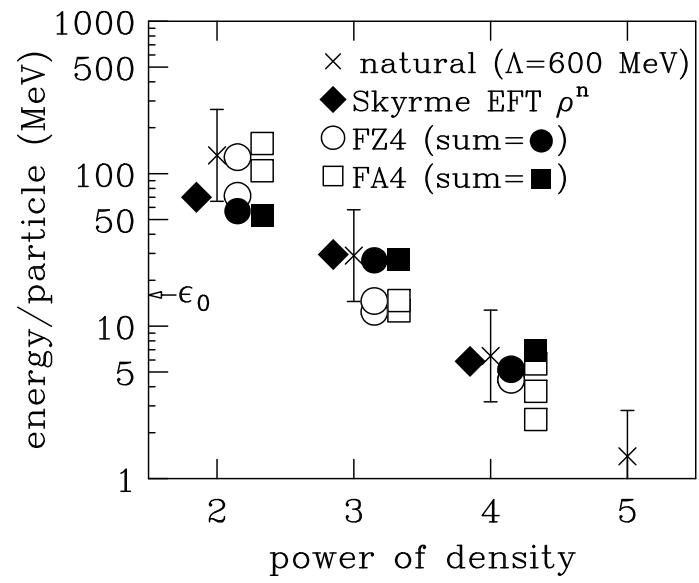

Figure 3: Energy contributions for a generalized Skyrme model compared to two QHD point-coupling models.

Finally, the EFT interpretation explains how to correctly account for QCD vacuum physics. Naturalness implies that the numerically important contributions from the vacuum are automatically incorporated into coefficients already in the model. In contrast, models based on a simple $\bar{N} N$ vacuum ("RHA") produce unnatural coefficients [2]. 


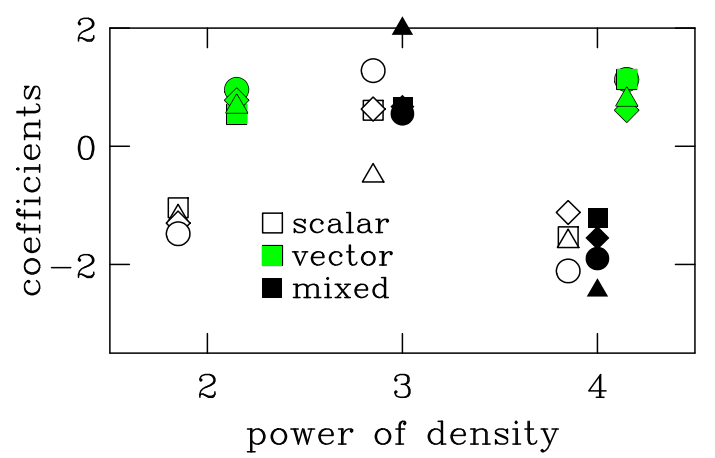

Figure 4. Coefficients for four good-fit QHD point-coupling models. Each model is represented by a different shape and the shading shows the type of term (scalar, vector, or mixed).

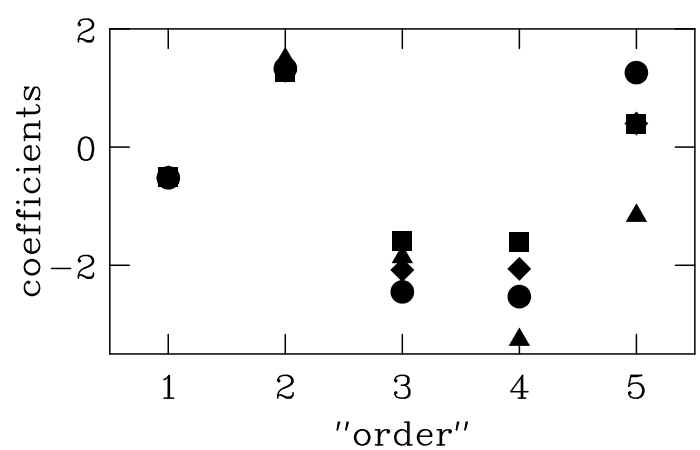

Figure 5. Optimal coefficients for the same four models as in Fig. 田. The "order" is determined by counting powers of $\rho_{+}$and $\rho_{-}$.

The "heavy" mesons $\omega, \rho$, and $\sigma$ that appear in QHD models have masses at the resolution scale $\Lambda$. The EFT perspective implies that we should be able to replace these meson interactions with local couplings between nucleons (so that the energy density has no heavy meson fields, but only powers of the scalar and vector densities) with similar success. This is indeed the case. In Fig. 8, a plot analogous to Fig. [1 is made for two general point-coupling models (labeled FZ4 and FA4). Results and conclusions similar to the QHD meson models are obtained (although one can truncate at third order and still find a good fit) [4].

As in Fig. 1, we see more than one QHD model with a good fit to nuclei, which implies

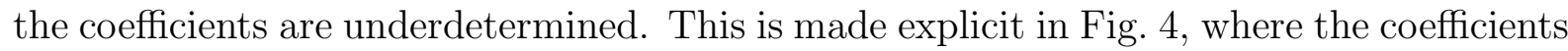
from four models show large variations even at leading order. (Note, however, that all coefficients are natural, i.e., order unity.) Can we find a more systematic power counting scheme? The similar size of the scalar density $\rho_{s}$ and the vector density $\rho_{\mathrm{B}}$ suggests that we count instead powers of $\rho_{+} \equiv\left(\rho_{s}+\rho_{\mathrm{B}}\right) / 2$ and $\rho_{-} \equiv\left(\rho_{s}-\rho_{\mathrm{B}}\right) / 2$. The variations of the new "optimal" coefficients for the four models are shown in Fig. 5. Leading orders are very well determined, with a systematic increase in uncertainty until even the sign is undetermined at the highest order shown.

These results suggest that a nonrelativistic point-coupling EFT, with an expansion in $\rho \equiv \rho_{\mathrm{B}}$ only, should work well for bulk nuclear observables. Indeed, the phenomenologically successful Skyrme models are of this type. Applying NDA shows that they are natural [5]. Since conventional Skyrme models are truncated at $\rho^{3}$, a generalized version with up to $\rho^{4}$ was fit to nuclei. The results in Fig. 3 show the same pattern predicted by NDA, except that the leading term is rather low. This is explained by comparing to the net results (filled symbols) from scalar and vector "two-body" contributions in the relativistic model: The underlying large mass scale is hidden by cancellations at order $\rho^{2}$. Note that higher orders follow the NDA predictions. A scale of $\Lambda=600 \mathrm{MeV}$ is consistent with the trends in all of the relativistic and nonrelativistic models.

What about the role of short-range correlations? Since we fit to observed nuclear properties, we do include correlation effects (approximately) in the coefficients of the model. An underlying assumption of our application of NDA to mean-field models is 
that the sizes of coefficients are dominated by the short-distance scales $\left(r \lesssim \Lambda^{-1}\right)$ and not potentially longer-ranged many-body scales. Or, at least, that the important manybody and short-distance scales are of similar magnitude. Density functional theory may provide a framework for the systematic inclusion of correlation effects [8]. Our mean-field models are analogs of the Kohn-Sham formalism, with local meson fields playing the role of (relativistic) Kohn-Sham potentials. Correlations are included exactly if the correct functional is used. Mean-field models approximate this functional with powers of fields or densities. The mean-field functional misses possible nonanalytic terms; a combination of EFT and density functional theory may show us how to systematically include them.

In summary, the application of EFT concepts and methods to mean-field models of nuclei reveals that:

- NDA provides an organizational principle at the mean-field level. The EFT power counting and the underdetermination of parameters by nuclei explain the success of conventional mean-field models [3].

- Mean-field models are approximate implementations of Kohn-Sham density functional theory, which means that correlation effects are included in simple Hartree calculations.

- Vacuum effects, chiral symmetry, and nucleon substructure are all included in general QHD models. This implies that the success of a particular non-QHD model is not necessarily evidence of the reality of the model dynamics.

- Ground-state nuclear properties provide information at low resolution. Models with different degrees of freedom (e.g., four- vs. two-component nucleons) are simply different organizations of the EFT. All are consistent with NDA.

Work is in progress to develop a more complete and consistent power counting, to connect to recent EFT studies of few-nucleon systems, and to develop density functional theory in an EFT framework. Explicit pion-loop corrections are being studied along with tests of general mean-field models for other observables, such as collective excitations, nuclear currents, and the isovector response.

\section{REFERENCES}

1. J.L. Friar, D.G. Madland and B.W. Lynn, Phys. Rev. C 53 (1996) 3085; J.L. Friar, Few Body Syst. 99 (1996) 1.

2. R.J. Furnstahl, B.D. Serot, H.-B. Tang, Nucl. Phys. A598 (1996) 539; A615 (1997) 441; A618 (1997) 446.

3. B.D. Serot and J.D. Walecka, Int. J. Mod. Phys. E6 (1997) 515.

4. J.J. Rusnak and R.J. Furnstahl, Nucl. Phys. A627 (1997) 455; A632 (1998) 607.

5. R.J. Furnstahl and J.C. Hackworth, Phys. Rev. C 56 (1997) 2875, and in preparation.

6. H. Georgi and A. Manohar, Nucl. Phys. B234 (1984) 189.

7. K. Saito and A.W. Thomas, Phys. Lett. B327 (1994) 9.

8. R.M. Dreizler and K.K.U. Gross, Density Functional Theory (Springer, Berlin, 1990). 